\begin{tabular}{|l|l|l|l|l|l|}
\hline Revista Praxis & ISSN: $1657-4915$ & Vol. 14 & No. 1 & $51-59$ & enero - junio de 2018 \\
\hline \multicolumn{6}{|c|}{ DOI: http://dx.doi.org/10.21676/23897856.2540 } \\
\hline
\end{tabular}

\title{
CARACTERÍSTICAS DE LOS MEJORES DOCENTES DE LOS PROFESORADOS EN EDUCACIÓN FÍSICA
}

\section{CHARACTERISTICS OF THE BEST TEACHERS IN THE PHYSICAL EDUCATION TEACHER TRAINING COLLEGES}

\author{
Sebastián Adolfo Trueba' ${ }^{1}$
}

\begin{abstract}
RESUMEN
El presente artículo analiza las características encontradas en un trabajo exploratorio realizado con el objetivo de hallar a los mejores docentes de los profesorados en Educación Física de la ciudad de Mar del Plata. Esta investigación se inscribe en una línea de investigación recientemente abierta en el Grupo de Investigación en Educación y Estudios Culturales (GIEEC) de la Facultad de Humanidades de la UNMdP y forma parte de la tesis doctoral del autor. Este trabajo comienza con una breve introducción con el fin de contextualizar la investigación, continúa con el desarrollo de la metodología utilizada y el análisis de los datos obtenidos, finalmente, se ofrecen algunas conclusiones provisorias.
\end{abstract}

Palabras clave: investigación biográfico-narrativa; profesores memorables; buena enseñanza; profesorado en educación física.

\section{ABSTRACT}

The present article analyzes the characteristics found in an exploratory work carried out to identify the best teachers in the physical education teachers' training colleges in the city of Mar del Plata. This research is part of a newly opened line of research in the Research Group on Education and Cultural Studies (GIEEC) of the Faculty of Humanities at The National University of Mar del Plata (UNMdP), and it is part of the doctoral thesis of the author. A brief introduction will be presented with the objective of providing a context to the research, followed by a development of the methodology and a later analysis of the data obtained. Finally, some provisional conclusions are offered.

Keywords: Narrative-Biographical Research; Memorable Teachers; Good Teaching; Physical Education Teacher Training College.

Fecha de recepción: junio 21 de 2017 / Fecha de aceptación: mayo 16 de 2018 / Publicado en línea: junio 20 de 2018 Tipología: Artículo de Investigación Científica y Tecnológica

Para citar éste artículo: Trueba, S. (2018). Características de los mejores docentes de los profesorados en Educación Física. Praxis, 14(1), 51-59. Doi: http://dx.doi.org/10.21676/23897856.2540

\footnotetext{
${ }^{1}$ Dr. en Humanidades y Artes con mención en Ciencias de la Educación (UNR). Grupo de Investigación en Educación y Estudios Culturales (GIEEC) dependiente del Centro de Investigaciones Multidisciplinarias en Educación (CIMED) de la Facultad de Humanidades, Universidad Nacional de Mar del Plata, Buenos Aires, Argentina. Correo electrónico: sebastiantrueba@gmail.com ORCID: 0000-0003-0011-5468
} 


\section{INTRODUCCIÓN}

L a enseñanza es uno de esos entornos humanos que raramente se beneficia de su pasado. Los grandes profesores aparecen, pasan por las vidas de sus estudiantes, y solo unos pocos de ellos quizás consigan alguna influencia en el vasto arte de la enseñanza. En la mayoría de los casos, su ingenio perece con ellos, y las siguientes generaciones deberán redescubrir de nuevo la sabiduría que dirigió sus prácticas (Bain, 2007, p.13).

El Grupo de Investigaciones en Educación y Estudios Culturales (GIEEC ${ }^{2}$ ) es un grupo de investigación que desarrolla sus actividades en la Universidad Nacional de Mar del Plata desde el año 2003 y que centra sus esfuerzos en la didáctica de la educación superior. A lo largo de los últimos quince años se desarrollaron investigaciones que abordaron cuestiones vinculadas a la Nueva Agenda de la Didáctica (Álvarez y Porta, 2004; Porta y Sarasa, 2006, 2008), a la narrativa en la enseñanza (Álvarez, 2006; Álvarez y Porta, 2008; Álvarez y Sarasa, 2007; Porta y Sarasa, 2006; Sarasa, 2008), las autobiografías profesionales de profesores memorables (Sarasa, Porta y Álvarez, 2008) y, a partir de allí, comenzaron a profundizar en el análisis de los mentores y prácticas docentes (Álvarez, Porta y Sarasa, 2010a, 2010b, 2010c), los grandes maestros, pasiones, identidades profesionales y la vida emocional y afectiva de dichos docentes memorables.

A partir de la trayectoria del autor en el programa de Doctorado en Humanidades y Artes con mención en Ciencias de la Educación de la Universidad Nacional de Rosario, se decidió presentar un proyecto de tesis que recupere las investigaciones desarrolladas a lo largo de más de una década en la Universidad Nacional de Mar del Plata pero incorporando un espacio aún no explorado: los profesorados en Educación Físi-

\footnotetext{
2 "EI GIEEC forma parte del Centro de Investigaciones Multidisciplinarias en Educación (CIMED) de la Facultad de Humanidades de la Universidad Nacional de Mar del Plata. Sus investigadores son profesores de varias disciplinas en distintos profesorados de dicha Facultad. Asimismo, se encuentran vinculados a la Carrera de Especialización en Docencia Universitaria, de la misma unidad académica" (Flores y Porta, 2013, p.32).
}

ca de la ciudad de Mar del Plata ${ }^{3}$. El proyecto de tesis doctoral se tituló de la siguiente manera: "Profesores memorables para los docentes formadores de los profesorados de Educación Física de la ciudad de Mar del Plata”. Como se puede interpretar a partir del título, una de las fases más importantes de la investigación radica en hallar a dichos profesores memorables. El presente trabajo describe esa búsqueda $y$ analiza los datos obtenidos.

\section{METODOLOGÍA}

Esta investigación es de corte interpretativo y se enfoca desde la perspectiva biográfico-narrativa (Bolívar, Domingo y Fernández, 2001). Se entiende que el enfoque interpretativo habilita miradas sistemáticas y rigurosas que no pretenden encontrar el valor de verdad, sino comprender e interpretar las prácticas humanas desde las tramas de significado que las originan y contienen. En el paradigma interpretativo, el conocimiento posee unas características que lo diferencian del conocimiento social nomotético formulado en leyes generalizables propias del positivismo. El conocimiento interpretativo es un conocimiento que está basado en las experiencias en el campo del investigador y de los participantes.

En tanto que la investigación biográfico-narrativa nos permite llegar a lugares a los que otros procedimientos no acceden, es por eso que se la utiliza en esta investigación, por su doble carácter de posibilidad y necesidad. En este sentido, esta perspectiva plantea un enfoque tan particular que influye en toda la investigación y no solo en las cuestiones metodológicas.

La investigación biográfico-narrativa, más allá de una mera metodología de recogida / análisis de datos, se ha constituido hoy en una perspectiva propia, como forma legítima de construir conocimiento en la investigación educativa. Como tal, defendemos que constituye un enfoque propio

\footnotetext{
${ }^{3}$ En Argentina la mayoría de los Profesorados de Educación Física se desarrollan por fuera del circuito universitario, en lo que se denominan Institutos Superiores de Formación Docente. En la ciudad de Mar del Plata los cuatro profesorados existentes son no universitarios, uno es de gestión estatal y tres son de gestión privada.
} 
(y no otra metodología "cualitativa" más), que altera algunos supuestos de la investigación sobre el profesorado y la enseñanza, así como el propio lenguaje de la investigación (Bolívar et al., 2001, p.10).

Durante la investigación se articularon distintos instrumentos que, en su complementariedad, posibilitaron profundizar en las historias de vida de los profesores memorables. Dichos instrumentos fueron:

- Cuestionarios narrativos, en los que los profesores de Educación Física que se desempeñaban en los profesorados seleccionaron a aquellos docentes que recordaban y reconocían como extraordinarios por su buena enseñanza. Además, fundamentaban su selección con frases breves y palabras que, según ellos, los caracterizaban.

- Entrevistas biográficas: el guión se construyó a partir del marco teórico y de los datos obtenidos con los cuestionarios narrativos. Una vez trascriptas se enviaron las a los entrevistados para que las corrigieran y enriquecieran. Una vez que las devolvieron se comenzó a trabajar con esos textos para construir el guión del grupo focal.

- Grupo focal: entrevista grupal en la que se reunió a los cuatro docentes del estudio y se profundizaron diversos conceptos surgidos en las entrevistas.

\section{Profesores memorables por su buena enseñanza}

Esta investigación se sostiene en el ideario de la Nueva Agenda de la Didáctica (Litwin, 1997, 2008) que, entre sus principios, contempla la visión positiva de la enseñanza, es decir, centrarse en los aspectos destacables de la didáctica en lugar de hacer referencia a lo reprochable; por ejemplo, en lugar de estudiar los errores o dificultades que más destacan los docentes formadores de los profesorados de educación física, ahondar en lo que hacen los mejores formadores en esta área. En ambos casos se abor- daría la formación docente, pero a partir de dos enfoques totalmente diferentes.

Lo fundamental, una vez aclarado lo anterior, es definir a qué se hace referencia con el concepto "docente memorable". Ken Bain (2007, 2012) es quien define por primera vez la categoría de profesor memorable o extraordinario como aquel que logra un éxito considerable en alentar los enfoques y los aprendizajes profundos con buena parte de su alumnado. Esta idea pone el acento en los resultados, en el aprendizaje. A pesar del tinte academicista de esta perspectiva, el autor también destaca lo siguiente:

No puedo hacer más hincapié en la simple pero magnífica noción de que la clave para comprender la mejor docencia no puede encontrarse en reglas o prácticas concretas, sino en las actitudes de los profesores, en su fe en la capacidad de logro de sus estudiantes, en su predisposición a tomar en serio a sus estudiantes y dejarlos que asuman el control sobre su propia educación, y en su compromiso en conseguir que todos los criterios y prácticas surjan de objetivos de aprendizaje básicos y del respeto y el acuerdo mutuo entre estudiantes y profesores (Bain, 2007, p.92).

Ante la necesidad de incorporar estos aspectos, se decidió tomar como referencia una definición que se ajuste más fielmente a la idea de docente memorable que nos interesa trabajar en la presente investigación, por lo tanto, se recuperó la que expresan Porta, Sarasa y Álvarez (2011), quienes califican como tales a aquellos que han dejado huellas en el recuerdo de sus alumnos por su buena enseñanza (Fenstermacher, 1989; Fenstermacher y Richardson, 2005). En este sentido, es posible, no solo

identificar prácticas específicas que conforman el quehacer habitual de los profesores memorables, sino también se trata de una cuestión de principios, creencias e interpretaciones sobre la enseñanza y el aprendizaje que se manifiestan en las formas de intervención que los profesores fomentan en sus clases. (Porta et al., 2011, p. 195)

La idea es hallar a estos profesores memorables de los profesorados de Educación Física de Mar del Plata, debido a lo cual se hizo necesario construir un 
criterio para determinar quiénes serían los sujetos que ingresarían a la investigación.

En este sentido, se consideró inicialmente que para que un profesor o profesora ingrese en la categoría de docente memorable, y se lo incorpore como uno de los sujetos de la investigación, este debía cumplir con los siguientes requisitos: en primera instancia, ser reconocido como docente memorable por sus propios colegas y ex-alumnos; en segunda instancia, sería necesario que, como mínimo, lo identificasen en esta categoría el $10 \%$ de los encuestados $y$, en el caso de que surgieran demasiados docentes caracterizados como memorables, se realizaría un corte en los tres sujetos más identificados en esta categoría, debido a las posibilidades de contactarlos, entrevistarlos y reunirlos para realizar un grupo focal; en caso de ser demasiados sujetos, disminuiría la posibilidad de concretar satisfactoriamente dichas etapas del trabajo de campo. Debido a lo cual, en primera instancia, se realizó un acercamiento a las instituciones que ofrecen esta carrera en la ciudad para encuestar a los docentes.

\section{Contextos institucionales / Decisiones metodológicas}

Una de las primeras decisiones metodológicas tomadas fue la de construir un criterio que permitiera que los docentes seleccionados como profesores memorables en este estudio fueran representativos de la muestra. Debido a lo cual se decidió encuestar a los profesores y profesoras de Educación Física que se desempeñan laboralmente en las instituciones formadoras. A dichos sujetos se les solicitó que completaran un cuestionario narrativo que contenía solamente tres preguntas. La primera solicitaba que se mencionaran los nombres de aquellos formadores de profesores que consideraban ejemplos de buena enseñanza; la segunda, que explicara con frases breves, el porqué de la elección en la pregunta anterior; y por último, que proporcionara cinco palabras clave que resumieran las características de la buena enseñanza de los docentes elegidos.

En la ciudad de Mar del Plata hay cuatro instituciones que ofrecen la carrera: "Profesorado en
Educación Física”, una de gestión estatal y tres de gestión privada, debido a lo cual se debió concurrir a todas ellas para solicitar autorización para encuestar a sus docentes.

Este proceso demandó bastante tiempo debido a que casi un centenar de profesores de educación física trabajan en estas carreras y el objetivo que se propuso fue el de suministrar estos cuestionarios narrativos a más del treinta por ciento de estos docentes, hombres y mujeres de las cuatro instituciones. Es necesario aclarar que algunos docentes dictan más de una materia y otros trabajan en más de una institución; finalmente, se obtuvieron 49 cuestionarios, lo que implica haber encuestado al $50 \%$ de los profesores y profesoras de educación física que trabajan en esta carrera.

\section{RESULTADOS}

Una vez obtenidas las encuestas, se las numeró con el fin de identificar cada una, ya que se trató de encuestas anónimas. Por lo que en ese momento la pregunta fundamental fue: ¿cómo organizar la información obtenida en la encuesta? En este sentido, no se utilizó un modelo construido por un tercero, sino que simplemente se realizaron tres trabajos diferentes, uno por cada pregunta.

El primero y más importante fue abrir un archivo de Excel en el que se creó una tabla con cada uno de los docentes mencionados como memorable en cada encuesta. Allí surgieron dos situaciones interesantes; la primera fue que muchos mencionaron a docentes fallecidos y en algunos casos expresaban que lo hacían como una especie de homenaje a pesar de saber que no entrarían en este estudio. Y el siguiente es que aparecieron menciones a algunos docentes que no son profesores de Educación Física, algo que no estaba presente en el planteo inicial. De hecho, el corte que originalmente estaba pensado en los tres docentes más representativos de esta categoría debió ampliarse a cuatro porque quedaron tres profesores de Educación Física y un docente que es Maestro Normal Nacional y Médico Pediatra. 
Sintetizando los hallazgos, fueron mencionados 75 docentes como memorables, 6 de los cuales ya habían fallecido, mientras que 8 provenían de otras disciplinas. El objetivo fue verificar quiénes eran los docentes que más se mencionaban en las encuestas como memorables por su buena enseñanza y seleccionar a los cuatro que más se repitieran; de ese modo, se habría cumplido con la primera parte de la investigación que se centraba en hallar a los sujetos que protagonizarían las siguientes etapas de la investigación.

Inicialmente, se debió organizar la información de manera provisoria, para pasar posteriormente al análisis profundo de la misma. A continuación, se describe este desarrollo primigenio de los datos. Por un lado, se abrieron cuatro carpetas en la computadora, cada una con la información correspondiente a cada docente memorable, en ellas se abrió un archivo Word con las frases breves que hacían referencia a cada sujeto seleccionado, es decir, que cada archivo de texto tenía como título "Frases breves del profesor xxx" y a continuación se transcribieron todas las frases indicando el número de cuestionario al que correspondía cada una.

Por último, se abrió un archivo de Excel en la carpeta de cada memorable, y allí se transcribieron todas las palabras que los encuestados usaron para describir a los sujetos de la investigación. Este paso supuso una complicación extra ya que se decidió organizar las palabras en categorías provisorias, por ejemplo, para un profesor se contabilizaron 64 palabras en total, pero la palabra compromiso se repite 7 veces y se la vinculó a palabras como pasión / apasionado (5 repeticiones), responsabilidad / responsable / puntualidad y presencia (5 repeticiones), dedicación / convicción / referente (4 repeticiones), y se las agrupó en una categoría provisoria denominada vínculo pedagógico. Estas agrupaciones fueron pensadas con el objetivo de obtener mayor cantidad de elementos a la hora de pasar a la segunda etapa del trabajo de campo y elaborar el guión de las entrevistas biográficas. A continuación, se detalla brevemente la información obtenida a partir de los cuestionarios narrativos, detallándolos por separado.

\section{Profesores memorables}

- De los 75 docentes mencionados, 12 aparecieron repetidos en dos encuestas, 4 en 3 , a 2 docentes se los mencionó 4 veces, 5 veces se mencionó a otros 4 , hubo 2 personas que obtuvieron 6 menciones, uno con siete y otro con 13. Debido a lo cual se seleccionaron a los cuatro con mayor cantidad de menciones, tres de los cuales son profesores de Educación Física y uno es Maestro Normal Nacional y Médico Pediatra.

- 6 docentes ya fallecidos que fueron mencionados en reconocimiento de su trayectoria.

- 67 profesores de Educación Física y 8 provenientes de otras disciplinas.

- Los porcentajes de menciones obtenidos por cada uno de los docentes seleccionados en relación a la cantidad de encuestas fue: $1^{\circ}$ ) 13 menciones $\left.(26,53 \%), 2^{\circ}\right) 7$ menciones $(14,29 \%), 3^{\circ}$ y $\left.^{\circ}\right) 6$ menciones $(12,24 \%)$.

- 17 mujeres $(22,7 \%)$ y 58 varones $(77,3 \%)$.

\section{Palabras que los caracterizan}

- 149 palabras para describir a los cuatro memorables.

- 16 veces aparece la palabra compromiso / comprometido y 15 veces se repite la palabra pasión / apasionado.

- En total se mencionan 98 palabras que se relacionan al vínculo pedagógico (compromiso, pasión, empatía, responsabilidad, convicción, entrega, dedicación, disposición, valoración del otro, buen tipo, ética, coherencia, carisma, humildad, humanismo, calidez humana, etc.)

- 37 palabras destacan la formación profesional (conocimiento, saber, conocimientos actualizados, excelencia, profesionalismo, investigación y capacitación permanente, etc.)

- 18 palabras refieren a cuestiones didácticas (planificación, motivador, estrategias didácticas, adaptación de contenidos, capacidad para transmitir, claridad, etc.) 
Por lo que se puede observar de la lectura de los puntos anteriores, surgieron tres categorías principales, relacionadas a tres cuestiones diferentes que de alguna manera se entrelazan: vínculo pedagógico (pasión, compromiso, ética, etc.) que sin duda es de gran importancia para la investigación debido a que, presumiendo que esto podría surgir, se planteó un enfoque interpretativo acerca de la influencia de los afectos y las emociones en las huellas que dejaron estos profesores memorables; formación profesional (conocimiento, actualización, formación, sabiduría, etc.), este es un aspecto que sin duda debía aparecer porque es impensado que los mejores docentes no se destaquen en las cuestiones disciplinares; y por último, cuestiones didácticas (planificación, motivador, claridad, capacidad para transmitir, etc.), esta categoría sin dudas surgió porque la investigación es de neto corte didáctico y se basa en la buena enseñanza. El análisis de estas tres categorías fue realizado en profundidad en etapas posteriores de la investigación; sin embargo, en esta primera aproximación se destacaron claramente.

\section{Frases breves}

Provisoriamente, se puede inferir lo mismo que surge con las palabras clave, pero expresado como parte de una narración. Por ejemplo: "Es muy humano y siempre está dispuesto a ayudarte y a aconsejarte. Explica todas las veces que necesites y todas con gran compromiso y pasión, incluso te presta sus propios libros. Siempre te alienta a que sigas adelante y a que seas mejor profesional". (C-1). O bien: "Por su humildad y principalmente por su formación humanística. Por no apuntar a la enseñanza memorística, sino a desarrollar habilidades del pensamiento y virtudes humanas. Apuntaron a desarrollar personas integrales, no técnicos”. (C-3, subrayado en el original).

Las incipientes categorías mencionadas anteriormente se ven potenciadas a partir de la incorporación de las frases breves y, además, son de gran utilidad para comprender el sentido que se les otorgan a las palabras clave y la coheren- cia de cada encuesta. Sin embargo, este ítem en particular tiene una intencionalidad doble que no poseen los dos anteriores; por un lado, lo expresado más arriba, pero por otro se buscó obtener un elemento que pueda triangularse más potentemente con otros instrumentos utilizados en las etapas posteriores de la investigación, como lo son la entrevista biográfica y el grupo focal. De esta manera, se pretende obtener una saturación de información que permita potenciar el análisis y las conclusiones finales de la tesis.

\section{DISCUSIÓN}

Se obtuvo la información requerida para la primera etapa de la investigación, es decir, hallar a los docentes memorables que serían los protagonistas de las siguientes etapas. El criterio original que se impuso para que un docente ingrese como memorable fue que tuviera como título de base Profesor de Educación Física y que fuera referenciado por lo menos por el 10 $\%$ de los encuestados. Ante la evidencia de que muchos encuestados mencionaban como profesores memorables a docentes provenientes de otras disciplinas, se decidió ampliar el criterio que refiere al título de base. Ahora bien, en realidad hubo cuatro docentes más que superaron el límite inferior del $10 \%$ de las menciones, llegando a un 10,20 \%, pero considerando que los sujetos de este estudio pasarían de ser cuatro a ocho se decidió no incluirlos debido a que dificultaría notablemente las etapas de las entrevistas y del grupo focal. De esta manera, quedó constituido el grupo por los cuatro formadores de formadores más representativos de la muestra.

Resultó sorprendente que para algunos de los encuestados el instrumento utilizado se constituyera en una forma de homenaje a los docentes que fallecieron, mencionándolos y, en algunos casos, sentándose con el encuestador para comentarle sus vivencias con dichos docentes. Otro factor a analizar radica en que poco más del $20 \%$ de los memorables seleccionados eran mujeres; y a pesar de que no se dispone del 
dato exacto de la cantidad de profesoras que trabajan en los profesorados en Educación Física, sin lugar a dudas superan ese porcentaje por lo que podría inferirse un sesgo machista que aparentemente aún perviviría en la formación docente. Esta cuestión no es pertinente para la investigación en curso, pero es destacable el dato como para abrir futuras líneas de investigación.

Las palabras que más veces se repitieron para definir a estos docentes memorables fueron: compromiso / comprometido y pasión / apasionado; ambos grupos de palabras resultan altamente significativos debido a que podría esperarse que aparecieran en mayor medida otros conceptos como: estudio, conocimiento, explicación, didáctica, etc. Esta situación nos da a entender que es correcto el enfoque que se realizó durante la investigación, lo que posiciona esta investigación en la Nueva Agenda de la Didáctica, la cual amplía su campo de análisis con respecto a la agenda clásica, y se sale de las cuestiones estrictamente vinculadas a la planificación, contenidos y evaluación; para incorporar lo social, político, económico, etc. Desde el posicionamiento clásico sería lógico que las palabras que más veces aparecieran en la encuesta fueran las mencionadas como esperables; sin embargo, el compromiso y la pasión abren el juego a un análisis mucho más profundo y humano de la didáctica.

A partir de lo anterior, se evidencia la necesidad de abordar con profundidad las cuestiones afectivas y emocionales tanto en el marco conceptual como en las siguientes etapas del trabajo de campo. Por otro lado, la coherencia entre las palabras sueltas y las frases breves que se utilizaron para describir a los docentes seleccionados sienta las bases para la construcción de los guiones, tanto de las entrevistas biográficas como del grupo focal. Al mismo tiempo permite inferir la concepción de buena enseñanza de los profesores de Educación Física encuestados. Los docentes fallecidos mencionados constituyen otra posible línea de investigación a futuro debido a que, evidentemente, han tenido una impronta demasiado grande como para olvidarla con el paso del tiempo.

\section{CONCLUSIONES}

La utilización de cuestionarios narrativos en una investigación de corte biográfico-narrativa resulta muy positiva porque puede aportar gran cantidad de datos tanto cuantitativos (hallar a los memorables a partir de la cantidad de menciones en la encuesta) como cualitativos (tipos de frases o palabras que describen a los memorables y que abren la posibilidad de desarrollar diferentes líneas en la investigación). Tomando solamente los datos obtenidos en la encuesta se pueden destacar provisoriamente algunas características que poseen aquellos docentes considerados como memorables por sus colegas y ex alumnos.

En primera medida, se debe destacar que existe un cierto consenso entre los formadores de formadores sobre quiénes son los mejores representantes de la buena enseñanza, es decir, existen características que destacan a un profesor extraordinario de otro que no lo es; esto queda reflejado a partir de que una buena parte del colectivo docente destaca a las mismas personas y por sus mismas cualidades, que se detallan a continuación:

- Los profesores memorables son reconocidos principalmente por los aspectos humanos y personales como su pasión, compromiso y ética, algo que se categorizó como vínculo pedagógico.

- También se destacan, aunque en menor medida, su formación profesional y su capacidad para enseñar.

- Estos tres aspectos (vinculares, profesionales y didácticos) sintetizan lo que los docentes de Educación Física que trabajan en los profesorados destacan como cualidades de los profesores memorables por su buena enseñanza.

Esto plantea también un desafío interesante para la formación de profesores en el área Educación Física, pues si las características antes 
descritas son las de los mejores profesores, al mismo tiempo deberían ser las que nos guíen para mejorar la formación de los futuros profesionales del área, de tal modo que podría desprenderse de esta primera aproximación, un horizonte formativo específico del área. Por tanto, la formación de Profesores en Educación Física debería apuntar primordialmente, a formar profesionales capaces de construir profundos vínculos pedagógicos; en segunda instancia, a docentes con conocimientos actualizados y con ganas de seguir aprendiendo y capacitándose; y, por último, con gran capacidad para enseñar, transmitir y motivar a sus alumnos.

Para finalizar el artículo, se desea destacar que a pesar de lo provisorio de los datos y del análisis de los mismos expresado en este trabajo, el ejercicio de reflexionar sobre la buena enseñanza e investigar sobre la didáctica en los profesorados de Educación Física se vuelve cada vez más necesario y apremiante para consolidar un campo tan potente como lo es el de las prácticas corporales educativas (Trueba, 2009a, 2009b). Este artículo intenta poner estos temas en debate a partir de la construcción de conocimiento científico y espera constituirse en un aporte que permita generar nuevas discusiones e investigaciones.

\section{REFERENCIAS BIBLIOGRÁFICAS}

Álvarez, Z. (2006). El discurso como unidad didáctica. En L. Porta y M. C. Sarasa (Eds.), Miradas críticas en torno a la formación docente y condiciones de trabajo del profesorado (pp. 245-266). Mar del Plata: GIEEC, Facultad de Humanidades, Universidad Nacional de Mar del Plata.

Álvarez, Z. y Porta, L. (2008). Biografías memorables, relatos sobre buenos profesores. En L. Porta y M. C. Sarasa (Eds.), Formación y desarrollo de la profesión docente en el profesorado: Las buenas prácticas y sus narrativas (pp. 219-245). Mar del Plata: GIEEC, Facultad de Humanidades, Universidad Nacional de Mar del Plata.

Álvarez, Z. y Porta, L. (Eds.). (2004). La formación del profesorado. Abordajes teóricos y miradas prácticas. Mar del Plata: GICIS, Facultad de
Humanidades, Universidad Nacional de Mar del Plata.

Álvarez, Z. y Sarasa, M. C. (2007). Una mirada a la buena enseñanza desde los relatos de profesores y alumnos. En M. Borgström y L. Porta (Eds.), La formación de profesores en Suecia y Argentina: lecturas y perspectivas convergentes (pp. 107-132). Edición bilingüe español-inglés. Mar del Plata: GIEEC, Facultad de Humanidades, Universidad Nacional de Mar del Plata.

Álvarez, Z., Porta, L. y Sarasa, M. C. (2010a). Itinerarios de la buena enseñanza a partir de los relatos biográficos docentes. Profesorado. Revista de Currículum y Formación del Profesorado, 14(3), 89-98.

Álvarez, Z., Porta, L. y Sarasa, M. C. (2010b). La recuperación biográfica del rol de los mentores como contribución a la formación de jóvenes docentes e investigadores. En II Congreso Internacional sobre profesorado principiante e inserción profesional a la docencia. El acompañamiento a los docentes noveles: prácticas y concepciones. INFOD, Buenos Aires, Argentina.

Álvarez, Z., Porta, L. y Sarasa, M. C. (2010c). Una exploración del rol de los mentores en las trayectorias profesionales de los buenos docentes universitarios. Revista Praxis Educativa, 14(14), 42-48.

Bain, K. (2012). Qué es la buena enseñanza. Revista de Educación, año 3(4), 63-74.

Bain, K. (2007). Lo que hacen los mejores profesores de Universidad. Barcelona: Universidad de Valencia.

Bolívar, A., Domingo, J. y Fernández, M. (2001). La investigación biográfico-narrativa en educación. Enfoque y metodología. Madrid: La Muralla.

Fenstermacher, G. (1989). Tres aspectos de la filosofía de la investigación en la enseñanza. En M. Wittrock (Ed.), La investigación en la enseñanza I. Enfoques, teorías y métodos. Barcelona: Paidós.

Fenstermacher, G. y Richardson, V. (2005). On Making Determinations of Quality in Teaching. Teachers' College Record, 107(1): 186-213.

Flores, G. y Porta, L. (2013). Urdimbre ética en la enseñanza universitaria desde la perspectiva de los estudiantes: conjunción de intelecto y afecto en los profesores memorables. Redex. Revista de Educación de Extremadura, (5), 29-49. 
Litwin, E. (2008). El oficio de enseñar. Condiciones y contextos. Buenos Aires: Paidós.

Litwin, E. (1997). El campo de la didáctica: la búsqueda de una nueva agenda. En: A. Camilloni, M. C. Davini, G. Edelstein, E. Litwin, M. Souto y S. Barco (Eds.), Corrientes didácticas contemporáneas (pp. 91-115). Bs. As.: Paidós.

Porta, L. y Sarasa, M. C. (Eds.). (2008). Formación $y$ desarrollo de la profesión docente en el profesorado: Las buenas prácticas y sus narrativas. Mar del Plata: GIEEC, Facultad de Humanidades, Universidad Nacional de Mar del Plata.

Porta, L. y Sarasa, M. C. (Eds.). (2006). Miradas críticas en torno a la formación docente $y$ condiciones de trabajo del profesorado. Mar del Plata: GIEEC, Facultad de Humanidades, Universidad Nacional de Mar del Plata.

Porta, L., Sarasa, M. C. y Álvarez, Z. (2011). Una experiencia en torno a la investigación sobre la enseñanza en el nivel superior. Revista de Educación, 2(3), 181-210.
Sarasa, M. C. (2008). En torno a los modelos de la buena enseñanza. En L. Porta y $\mathrm{M}$. C. Sarasa (Eds.) Formación y desarrollo de la profesión docente en el profesorado: Las buenas prácticas y sus narrativas (pp. 183-217). Mar del Plata: GIEEC, Facultad de Humanidades, Universidad Nacional de Mar del Plata.

Sarasa, M. C., Porta, L. y Álvarez, Z. (2008). Estudio de la buena enseñanza universitaria: una contribución a la didáctica de la educación superior. En Actas Encuentro de Universidades Latinoamericanas, (CD-ROM). Mar del Plata: Secretaría de Extensión, UNMDP.

Trueba, S. (2009a). Teoría de los campos y campo de las prácticas corporales educativas. Revista Ser Corporal, (1), 41-55. ISSN 1852-1878.

Trueba, S. (2009b). Implicancias pedagógicas y didácticas de los profesores de educación física en el Campo de las Prácticas Corporales Educativas. Revista Ser Corporal, (2), 27-32. ISSN 1852-1878. 